\title{
Editorial
}

\section{Nurse led, multidisciplinary intervention in chronic heart failure}

Angiotensin converting enzyme (ACE) inhibitors reduce morbidity and mortality in chronic heart failure $(\mathrm{CHF})$, however, the prognosis for patients with this disorder remains poor. In the treatment arm of the studies of left ventricular dysfunction (SOLVD) $35 \%$ of patients in the enalapril group died within the 3.5 years of follow up, $46 \%$ were admitted to hospital with worsening $\mathrm{CHF}$, and $69 \%$ were admitted to hospital for any reason. ${ }^{1}$ Digoxin may reduce the need for admission ${ }^{2}$ but hospitalisation remains a frequent, distressing, costly, and recurring consequence of $\mathrm{CHF}^{3}$.

A number of investigators have attempted to identify potential precipitating and contributing factors to hospital admissions in patients with $\mathrm{CHF}^{4-11}$ The report by Michalsen et al is an important contribution to this serious and costly health issue. ${ }^{12}$ Several common themes have emerged: limited understanding of the $\mathrm{CHF}$ state and its treatment is almost universal among patients; poor adherence to diet and pharmacological treatment is a frequent problem; other avoidable factors commonly contribute to $\mathrm{CHF}$ decompensation - for example, respiratory infections (preventable by immunisation), poor control of hypertension; and inadequate discharge planning and follow up.

Recently, the concept that specialised and intensive follow up of these often elderly and vulnerable patients, aimed at correcting the aforementioned deficiencies in care, might improve outcome and reduce hospitalisation has been tested in two important studies. The first landmark study in this area was by Rich et al. ${ }^{13}$ These authors conducted a prospective, randomised trial of the effect of a nurse directed, multidisciplinary intervention on the rates of hospital readmission, quality of life, and costs of care within 90 days of discharge among elderly, high risk patients who were hospitalised for CHF. The intervention consisted of comprehensive education of the patient and family, a prescribed diet, social service consultation and planning for an early discharge, a review of medications, and intensive home and clinic based follow up with frequent telephone contact. ${ }^{13-15}$ The principal goals of follow up were to reinforce the patient's education, ensure adherence to diet and medications, and identify recurrent symptoms amenable to treatment on an outpatient basis. Survival for 90 days without readmission was achieved in 91 of $142(64 \%)$ of patients in the intervention group compared to 75 of $140(54 \%)$ in the control group $(p=0.09)$. There were 94 readmissions in the control group and 53 in the intervention group (risk ratio 0.56, $\mathrm{p}=0.02$ ). The number of readmissions for $\mathrm{CHF}$ was reduced by $56 \%$ in the treatment group ( $24 v 54$ in the control group; $p=0.04)$. Fewer intervention group patients had more than one readmission ( $9 v 23$; risk ratio $0.39, p=0.01)$. Quality of life was significantly better in the intervention group $(p=0.001)$ and the overall cost of care was $\$ 460$ less per patient in this group. ${ }^{13}$

These impressive results have been replicated in another study. Stewart et al, as part of a larger clinical trial, ${ }^{16}$ randomised hospitalised CHF patients with impaired left ventricular systolic function, exercise intolerance, and a history of at least one admission for acute heart failure to usual care $(\mathrm{n}=48)$ or a home based intervention $(\mathrm{n}=49) .{ }^{17}$ The home based intervention comprised a single home visit one week after discharge by either a nurse or a pharmacist. The purpose of this visit was to optimise medication management, identify early clinical deterioration, and intensify medical follow up/caregiver vigilance where appropriate. The primary end point of the study was the frequency of unplanned readmission plus out of hospital death within six months of discharge. Home based intervention patients had both fewer unplanned readmissions (36 $v 63 ; \mathrm{p}=0.03)$ and out-of-hospital deaths ( $1 v 5$; $\mathrm{p}=0.11$ ), equating to a mean (SD) of $0.76(0.91) v 1.4$ (1.8) events per patient in the usual care and home based intervention groups, respectively $(p=0.03) .{ }^{16} 17$ Home based intervention patients also had fewer days of hospitalisation (261 $v 452 ; \mathrm{p}=0.05)$ and fewer multiple $(\geqslant 3)$ readmissions for CHF $(p=0.02) .{ }^{16}{ }^{17}$

In this issue a third report by Cline et al adds some further evidence in favour of nurse led multidisciplinary intervention following admission to hospital with $\mathrm{CHF}^{18}$ These authors randomised 206 patients aged 65-84 years hospitalised with heart failure to intervention by specially trained nurses or to usual care. The special intervention included an educational programme for patients and their families, concentrating on treatment. Guidelines on adjusting treatment in response to sodium and water overload and fluid depletion were also provided. This programme was carried out over two 30 minute visits to the patient in hospital and a one hour visit to the patient and family two weeks after discharge. A special diary was provided. Close, easily accessible, patient initiated follow up was provided at a nurse run, hospital based clinic and through telephone contact. One year mortality did not differ between groups. Time to first readmission over the same period however was $33 \%$ longer in the intervention group (106 $v 141$ days; $\mathrm{p}<0.05)$.

The mean number of hospitalisations was $36 \%$ lower (1.1 $v 0.7 ; \mathrm{p}=0.08)$ and the total days hospitalised $49 \%$ lower $(8.2 v 4.2 ; \mathrm{p}=0.07)$ in the intervention group. Total annual costs of care also tended to be lower in the intervention group (US $\$ 2294 v 3594 ; \mathrm{p}=0.07$ ). It should be noted that this study recruited patients with a much lower rate of readmission than those in the previous two studies.

In an earlier report, Kornowski et al also reported that home based intervention by physicians reduced hospitalisation rates and improved quality of life in a group of elderly patients with $\mathrm{CHF}^{19}$ This analysis, however, was not a randomised controlled trial, instead comparing hospitalisation rates and quality of life in the year before home based intervention to the first year of the home care programme. Nevertheless, this study does support the findings of Rich et al and Stewart et al.

It seems clear that specialist nurse led intervention can improve the dismal morbidity of CHF. All three randomised trials involved predischarge education and home visiting after discharge. The first two state that they explicitly emphasised the important of adherence to treatment 
and the early detection and treatment of clinical decompensation, suggesting that these were key elements in the success of these programmes. Despite the clear benefits of adopting this type of programme, a number of important issues regarding implementation and achieving optimal cost-benefit are yet to be determined.

- What is the optimal timing, intensity, and duration of such interventions? Rich et al used an intensive programme that lasted three months after hospitalisation. In contrast Stewart et al used a less intensive (and potentially most cost-effective) programme and yet both were associated with significantly fewer readmissions. Cline et al had a nurse clinic visit at eight months after discharge and offered consultations with a cardiologist at one and four months.

- What is the duration of effect of these programmes? Neither of the first two studies extended follow up beyond six months and their longer term effects are therefore yet to be determined.

- What are the exact mechanisms of beneficial effect? Although there is evidence of improved compliance following this type of intervention ${ }^{15}$ it is invariably difficult to determine the precise effect(s) of multifactorial interventions; such a determination has major implications for the first two issues.

- Are these programmes associated with improved survival? None of the three randomised studies were sufficiently large or prolonged to detect a difference in mortality and yet two reported a trend towards reduced mortality during study follow up among patients exposed to their respective interventions. One might question, however, whether reduction in mortality is an important issue in these chronically ill, elderly, patients. A number of studies examining these issues are currently in progress and will hopefully shed more light on how to optimise this type of programme.

Comparable interventions in other settings or other models of care may hold similar promise. ${ }^{20-24} \mathrm{~A}$ number of recent reports suggest that specialist heart failure clinics may reduce the frequency of emergency hospital admissions in patients with $\mathrm{CHF}^{20-24}$ Unlike the heart failure studies, these reports relate to relatively young patients, usually referred for transplant evaluation. Furthermore these observations are not based on randomised studies, relying instead on comparisons of admission rates before and after referral or before and after setting up of the clinic. Furthermore, while two of the programmes involved combined nurse and physician follow up, one was nurse led ${ }^{22}$ and the other physician based. ${ }^{20}$ The latter also involved a period of inpatient treatment adjustment according to invasive haemodynamic measurements.

In summary, it seems clear that we can do more for patients with CHF. The key seems to be spending time on education, especially in relation to medication. Reinforcement is necessary. Simplification of drug regimens and adherence counselling appear vital. Early treatment adjustment in response to signs or symptoms of deterioration is also important. Ease of contact with health professionals may be an important aspect of any programme to help patients with CHF. Programmes involving these principles applied to elderly patients admitted to hospital with $\mathrm{CHF}$ are of proved worth. Nurse leadership of such programmes has also been successful. The benefits are large: reduced hospital admission rates, improved quality of life, and cost savings. Subject to the fine tuning of the optimal nature, timing, and duration of these programmes, we need to know whether similar interventions can be extended to other patient groups with CHF (for example, outpatient clinic populations) and patients with other chronic cardiac problems.

J J V MCMURRAY

Consultant Cardiologist

Department of Cardiology,

Western Infirmary,

Glasgow G11 6NT, UK

e-mailj.mcmurray@bio.gla.ac.uk

S STEWART

Senior Cardiac Research Nurse

The Queen Elizabeth Hospital,

South Australia 5011, Australia

1 The SOLVD Investigators. Effect of enalapril on survival in patients with reduced left ventricular ejection fractions and congestive heart failure. $N$ Engl f Med 1991;325:293-302.

2 The Digitalis Investigation Group. The effect of digoxin on mortality and morbidity in patients with heart failure. N Engl f Med 1997;336:525-533.

3 McMurray J, Davie A. The pharmacoeconomics of ACE inhibitors in heart failure. Pharmacoeconomics 1996;9:188-97

4 Opasich C, Febo O, Riccardi PG, et al. Concomitant factors of decompensation in chronic in heart failure. Am f Cardiol 1996;78:354-7.

5 Antonios TFT, MacGregor GA. Salt-more adverse effects. Lancet 1996;348:250-1

6 Nikolaus T, Spechtleible N, Kruse W, et al. Early rehospitalization of very elderly patients - causes and prevention. Deutsche Medizinische Wochenelderly patients-Cause
schrift 1992;117:403-7.

7 Vinson JM, Rich MW, Sperry JC, et al. Early readmission of elderly patients with congestive heart failure. F Am Geriatr Soc 1990;38:1290-5.

8 Ghali JK, Kadakia S, Cooper R, et al. Precipitating factors leading to decompensation of heart failure. Arch Intern Med 1988;148:2013-16.

9 Chin MH, Goldman L. Factors contributing to the hospitalization of patients with congestive heart failure. Am f Public Health 1997;87:643-8.

10 Chin MH, Goldman L. Correlates of early hospital readmission or death in patients with congestive heart failure. Am f Cardiol 1997;79:1640-4.

11 Wagdi P, Vuilliomenet A, Kaufmann U, et al. Ungenügende Behandlungsdisziplin, Patienteninformation und Medikamentenverschreibung als Ursachen fur die Notfallhospitalisation bei chronisch herzinsuffizienten Patienten. Schweiz Med Wschr 1993;123:108-12.

12 Michalsen A, König, Thimme W. Preventable causative factors leading to hospital admission with decompensated heart failure. Heart 1998;80:43741.

13 Rich MW, Beckham V, Wittenberg C, et al. A multidisciplinary intervention to prevent the readmission of elderly patients with congestive heart failure. NEngl F Med 1995;333:1190-5.

14 Rich MW, Vinson JM, Sperry JC, et al. Prevention of readmission in elderly patients with congestive heart failure - results of a prospective, randomized pilot study. $\mathcal{F}$ Gen Intern Med 1993;8:585-90

15 Rich MW, Gray DB, Beckham V, et al. Effect of a multidisciplinary intervention on medication compliance in elderly patients with congestive heart failure. Am F Med 1996;101:270-6.

16 Stewart S, Pearson S, Luke CG, et al. Effects of home-based intervention on unplanned readmissions and out-of-hospital deaths. I Am Geriatr Soc 1998;46:174-80

17 Stewart S, Pearson S, Horowitz JD. Effects of a home-based intervention among congestive heart failure patients discharged from acute hospital care. Arch Intern Med 1998;158:1067-72.

18 Cline CMJ, Israelsson BYA, Willenheimer RB, et al. A cost effective management programme for heart failure reduces hospitalisation. Heart 1998;80:442-6.

19 Kornowski R, Zeeli D, Averbuch M, et al. Intensive home-care surveillance prevents hospitalization and improves morbidity rates among elderly prevents hospitalization and improves morbidity rates among elderly

20 Fonarow GC, Stevenson LW, Walden JA, et al. Impact of a comprehensive heart failure management program on hospital readmission and functional status of patients with advanced heart failure. $\mathcal{F} \mathrm{Am}$ Coll Cardiol 1997;30:725-32.

21 Smith LE, Fabbri SA, Pai R, et al. Symptomatic improvement and reduced hospitalization for patients attending a cardiomyopathy clinic. Clin Cardiol 1997;20:949-54

22 West JA, Miller NH, Parker KM, et al. A comprehensive management system for heart failure improves clinical outcomes and reduces medical resource utilization. Am f Cardiol 1997;79:58-63.

23 Reis SE, Holubkov R, Edmundowicz D, et al. Treatment of patients admitted to the hospital with congestive heart failure: speciality-related disparities in practice patterns and outcomes. I Am Coll Cardiol 1997;30:733-8.

24 Hanumanthu S, Butler J, Chomsky D, et al. Effect of a heart failure program on hospitalization frequency and exercise tolerance. Circulation 1997;96: 2842-8. 